\title{
Isolation and Characterization of Extraocular Muscle-Derived Muscle Progenitor Cells from Normal and Graves' Orbitopathy Patients
}

\author{
Mira Park, ${ }^{1}$ Sarmila Nepali, ${ }^{2}$ and Helen Lew ${ }^{1}$
}

\begin{abstract}
Mesenchymal stem cells (MSCs) are useful for various purposes, including tissue engineering, regeneration, and gene therapy. MSCs isolated from extraocular muscles (EOMs) can be easily expanded in vitro, and can undergo multilineage differentiations involving adipogenesis, chondrogenesis, osteogenesis, and even neuronal or myogenic differentiation. This study aimed to isolate, characterize, and compare extraocular muscle-derived muscle progenitor cells (EOM-MPCs) from normal subjects and patients with Graves' orbitopathy (GO). EOM was obtained during strabismus surgery. Flow cytometry was conducted to identify CD surface antigens such as CD34, CD45, CD44, CD59, CD73, and CD90. We quantitated various cytokines secreted from MSCs, including interleukin (IL)-1 $\alpha$, IL-2, IL-6, IL-8, IL-10, IL-12, IL17A, tumor necrosis factor (TNF)- $\alpha$, and interferon (IFN)- $\gamma$, using a multi-analysis enzyme-linked immunosorbent assay array kit. We performed Oil Red O staining for adipogenesis, Alzarin Red staining for osteogenesis, Alcian blue staining for chondrogenesis, and polymerase chain reaction to measure messenger RNA expression during myogenesis. Our results show that EOM-MPCs from normal subjects and GO patients had similar levels of surface antigen expression and cytokine secretion. There was also no significant difference in the multilineage differentiation of adipocytes, chondrocytes, osteocytes, and myoblasts from EOM-MPCs taken from normal subjects and GO patients. However, hyaluronic acid synthetase 2 expression was higher after induction with tafluprost in EOM-MPCs from GO patients when compared with normal subjects. Together, these results show that EOM-MPCs derived from normal subjects are a good source for stem cell-based therapy for various disorders.
\end{abstract}

Keywords: adipogenesis, chondrogenesis, muscle-derived stem cells, myogenesis, osteogenesis

\section{Introduction}

$\mathrm{M}$ ESENCHYMAL STEM CELLS (MSCs) originating from adipose tissue, placenta, umbilical cord blood (UCB), and bone marrow can differentiate from mesenchymal cell lineages into adipogenic, osteogenic, and chondrogenic cells. They can function with relatively high efficiency and can effectively repair tissues in vivo [1]. The major areas of cell therapy involve conditions such as tissue repair and regeneration, and gene therapy [2].

When embryonic or adult stem cells for tissue-specific repair are converted, they can differentiate, in vivo, into unnecessary cell lineages. Furthermore, adult multipotent cells induced to differentiate into a single cell type can differentiate into other cell types [3].

Pacheco-Pinedo et al. [4] reported that extraocular muscle (EOM) contains many stem cells when compared with limb muscles, and that it exhibits high proliferative capacity.
Stem cells have been recently isolated from EOM tissue, and their multipotent differentiation capabilities have been investigated. The stem cell components of EOM are classified by their ability to exclude Hoechst 33342 dye [5]. Human EOM-derived MSCs can be easily expanded in vitro and can undergo multilineage differentiation, indicating that they are a novel source of mesenchymal cells for regenerative therapy [3].

Graves' orbitopathy (GO) is caused by autoantibodymediated inflammatory and fibrotic events in EOM, resulting in restrictive strabismus and diplopia [2]. The key feature of EOM enlargement in GO is the accumulation of glycosaminoglycans, including hyaluronic acid (HA) and collagen fibrils [6]. Edematous swelling and subsequent fibrosis of EOM lead to expansion of the orbital volume, followed by detrimental GO symptoms such as double vision, loss of vision, proptosis, corneal ulceration, and even blindness [2].

\footnotetext{
${ }^{1}$ Department of Ophthalmology, CHA Bundang Medical Center, CHA University, Seongnam, Republic of Korea.

${ }^{2}$ Department of Ophthalmology, University of Miami, Coral Gables, Florida.
} 
Regarding skeletal muscle repair after injury, myogenic progenitor cells migrate, differentiate, proliferate, and integrate with surrounding muscle tissues during regeneration [7]. Stromal fibroblasts infiltrate the wound, secrete growth factors and cytokines, and deposit fibrotic extracellular matrix components like collagen I and fibronectin after severe damage [8]. Because scar tissue formation usually interferes with regeneration, only a portion of the wound area completes repair without fibrosis [8]. However, the processes involved in successful repair, including the recruitment of myoblasts and secretion of fibroblast factors, are still under study.

Goetsch et al. [9] reported a method to simultaneously isolate myoblasts and fibroblasts from single muscle tissue, and they established an in vitro co-culture wound healing assay. They optimized the protocol to efficiently sort primary fibroblasts and myoblasts by serial preplating, followed by the cultivation of both cell types. This study therefore aimed to isolate, characterize, and compare the EOM-derived muscle progenitor cells (EOM-MPCs) of normal subjects and GO patients. We isolated EOM-MPCs and investigated their differentiation potential and gene expression profiles.

\section{Materials and Methods}

This study was reviewed and approved by Institute Review Board Committee (IRB) of Bundang CHA Medical center, CHA University, Seongnam, South Korea. The study followed the tenets of the Declaration of Helsinki and written informed consent was obtained from each patient.

\section{Subjects}

EOM tissues were obtained from two patients (men, $17.5 \pm 13.5$ years) with alternating exotropia and two GO patients (women, 49.0 \pm 1.0 years) with restrictive strabismus during the strabismus surgery.

\section{EOM tissue collection}

EOM samples were obtained from patients undergoing corrective surgery for strabismus in collaboration with the Department of Ophthalmology at Bundang CHA Medical Center after written informed consent and in accordance with the hospital human ethics committee guidelines.

\section{Isolation of EOM-MPCs}

The muscle progenitor cells (MPCs) were isolated following the protocol [9]. In brief, muscle pearls were microdissected and washed in phosphate-buffered solution (PBS; Welgene, Daegu, South Korea) to remove erythrocytes, micro-dissected and digested with $0.25 \mathrm{mg} / \mathrm{mL}$ collagenase (Thermo Fisher Scientific, Waltham, MA) at $37^{\circ} \mathrm{C}$ for $60 \mathrm{~min}$ under shaking constantly. After removal of supernatant, cells were suspended in primary media containing Ham's F-10 (Thermo Fisher Scientific) with $20 \%$ fetal bovine serum (FBS; Thermo Fisher Scientific) and $1 \%$ penicillin-streptomycin solution (P/S; Thermo Fisher Scientific) supplemented with $50 \mu \mathrm{g} / \mathrm{mL}$ gentamicin (Sigma-Aldrich, St. Louis, MO) and $2.5 \mathrm{ng} / \mathrm{mL}$ recombinant human fibroblast growth factor-4 (hFGF4; Peprotech, Inc., Rocky Hill, NJ). Next, the collagenased tissues were transferred to new dish by time course. At the last step, the collagenased tissues were discarded and cells were grown in collagen-coated plate in enrichment media containing 1:1 Ham's F-10 and Dulbecco's modified Eagle medium (DMEM), 20\% FBS, and 1\% P/S for 7-10 days in $5 \% \mathrm{CO}_{2}$ and $37^{\circ} \mathrm{C}$. Cells were passaged when it reached $60 \%-80 \%$ confluence with media changed in 2- to 3-day interval. After passage 0, cells were grown in growth media containing DMEM/F12, $10 \% \mathrm{FBS}$, and $1 \% \mathrm{P} / \mathrm{S}$ for further passages.

\section{Flow cytometry}

Cells at passage 3 (P.3) were detached by using trypsinEDTA $0.2 \%$ (Thermo Fisher Scientific) and fixed with methanol for $10 \mathrm{~min}$ at $-20^{\circ} \mathrm{C}$, washed with $1 \%$ bovine serum albumin (BSA) in PBS and then incubated with permeabilization buffer: $0.1 \%$ Triton $\mathrm{X}-100,1 \%$ BSA in PBS for $10 \mathrm{~min}$ at $4^{\circ} \mathrm{C}$ [10]. Cells were incubated with antibodies raised against CD34, CD45, CD44, CD59, CD73, and CD90 (Santa Cruz Biotechnology, Dallas, TX) on ice for $30 \mathrm{~min}$. Cells were pelleted, washed, and fixed in $1 \%$ paraformaldehyde. Fluorescence-activated cell sorting analysis was performed on CytoFLEX flow cytometer (Beckman Coulter, Brea, CA) at low flow rate and 10,000 events. Data were analyzed with CytoExpert analysis software (Beckman Coulter).

\section{Multi-analyte ELISArray}

We quantified various cytokines and growth factors concentrations secreted from EOM-MPCs using Multi-analyte ELISArray kit (Qiagen, Hilden, Germany) according to the manufacturer's instructions. This assay kit measured protein concentrations of interleukin (IL)-1 $\alpha$, IL-2, IL-6, IL-8, IL-10, IL-12, IL-17A, tumor necrosis factor (TNF)- $\alpha$, and interferon (IFN)- $\gamma$. Absorbance was measured using VersaMax ${ }^{\mathrm{TM}}$ Plus Rom v1.23 enzyme-linked immunosorbent assay (ELISA) plate reader (Molecular Devices ${ }^{\circledR}$, Sunnyvale, CA) at $450 \mathrm{~nm}$.

\section{Adipogenic differentiation and Oil Red O staining}

EOM-MPCs were cultured with adipogenic medium that contains $30 \mu \mathrm{M}$ indomethacin, $5 \mu \mathrm{M}$ insulin, $0.5 \mathrm{mM} \mathrm{2-}$ isobutyl-1-methylxanthine, and $1 \mu \mathrm{M}$ dexamethasone (SigmaAldrich) $[10,11]$. Medium was changed every 2-3 days. At 14 days of differentiation, cells were fixed with $10 \%$ neutral formalin for $1 \mathrm{~h}$ and stained with Oil Red $\mathrm{O}$ solution for $30 \mathrm{~min}$. For quantitation of lipid vacuoles, cells were extracted with $200 \mu \mathrm{L}$ of $100 \%$ isopropanol (Merck, Darmstadt, Germany) for 15 min and absorbance measured using VersaMax Plus Rom v1.23 ELISA plate reader (Molecular Devices) at $540 \mathrm{~nm}[12]$.

\section{Osteogenic differentiation and Alizarin Red S staining}

EOM-MPCs with confluent growth were cultured in an osteogenic differentiation medium containing DMEM, 10\% FBS, $1 \%$ P/S, $10 \mathrm{nM}$ dexamethasone (Sigma-Aldrich), $60 \mu \mathrm{M}$ ascorbic acid (Sigma-Aldrich), and $10 \mathrm{mM} \beta$ glycerophosphate disodium (Cayman Chemical Company, Ann Arbor, MI) [11,13]. Cells were cultured in complete growth medium (control) or induced medium for 2 weeks, medium was changed every 2-3 days. Cells were stained with Alizarin Red S staining (Sigma-Aldrich), and mineralization 
Table 1. Lists of Human Primers Sequences Used for Polymerase Chain Reaction

\begin{tabular}{|c|c|c|}
\hline Primers & Forward sequences & Reverse sequences \\
\hline DESMIN & CCAACAAGAACAACGACG & TGGTATGGACCTCAGAACC \\
\hline MYOSTATIN & CTGTAACCTTCCCAGGACCA & CCСАTCCAAAAGCTTCAAAA \\
\hline IGFBP5 & CAACCTGGGCCTTGTACTGT & AGGAGGCCAGAGTCTGTGAA \\
\hline$P P A R \gamma$ & ATTGACCCAGAAAGCGATTC & ATTGACCCAGAAAGCGATTC \\
\hline$C / E B P \alpha$ & GGGTCTGAGACTCCCTTTCCTT & CTCATTGGTCCCCCAGGAT \\
\hline FABP4 & AACCTTAGATGGGGGTGTCCTG & TCGTGGAAGTGACGCCTTTC \\
\hline$O C N$ & CCCAGGCGCTACCTGTATCAA & GGTCAGCCAACTCGTCACAGTC \\
\hline$B M P 2$ & TTTGGACACCAGGTTGGTGAA & ACGAATCCATGGTTGGCGT \\
\hline SP7 & GCACAAACATGGCCAGATTC & AGA AATCTACGAGCAAGGTC \\
\hline COL1A & TCC TGCCGATGTCGCTATC & CAAGTTCCGGTGTGACTCGTG \\
\hline ACAN & ССТССССТTCACGTGTAAAA & GCTCCGCTTCTGTAGTCTGC \\
\hline SOX9 & TACCCGCACTTGCACAAC & TCTCGCTCTCGTTCAGAAGTC \\
\hline PАХЗ & TGCAGTGATGTTTGCCATTT & GTGCCTACCTCATTCGTGGT \\
\hline MYODI & CGCTCCAACTGCTCCGACGG & GACACCGCCGCACTCTTCCC \\
\hline MYOGENIN & GAGCTCACCCTGAGCCCCGA & GCAGGCACTGGCCTGGACAG \\
\hline DYSTROPHIN & TTCTCAGCTTATAGGACTGCC & GGAGTGCAATATTCCACCAT \\
\hline HAS1 & AAACTGCTGCAAGAGGTTATTCCT & GACTCCTGGGTCAGCTTCCTAAG \\
\hline HAS2 & CTATGCTTGACCCAGCCTCATC & ACACTGCTGAGGAATGAGATCCA \\
\hline TSG6 & ATGTCCATGGCTTTGTGGGAA & ATTTGGAAACCTCCAGCTGT \\
\hline TSHreceptor & ATCAGGAGGAGGACTTCAGA & TTTGAGGGCATCAGGGTCTA \\
\hline 18s rRNA & TGAGAAACGGCTACCACATC & ACTACGAGCTTTTTAACTGC \\
\hline
\end{tabular}

during osteogenesis was measured quantitatively following manufacturer's instructions. In brief, cells were incubated with $10 \%$ acetic acid for $30 \mathrm{~min}$ at room temperature (RT), scraped, and transferred to new tubes, followed by heating at $85^{\circ} \mathrm{C}$ for $10 \mathrm{~min}$ and then cooled in ice. After removal of debris by centrifugation, supernatant was transferred to new tube, normalized with $10 \%$ ammonium hydroxide solution, and absorbance measured at $405 \mathrm{~nm}$.

\section{Chondrogenic differentiation and Alcian blue staining}

EOM-MPCs with $80 \%$ confluence were cultured in chondrogenic differentiation medium containing DMEM/ F12, $10 \%$ FBS, $1 \%$ P/S supplemented with $10 \mathrm{ng} / \mathrm{mL}$ recombinant transforming growth factor beta (TGF- $\beta$ ), $50 \mu \mathrm{M}$ ascorbic acid, and $6.25 \mu \mathrm{g} / \mathrm{mL}$ insulin (TAI medium) [11] in 24-well plates. After 2 weeks of differentiation, cells were stained with Alcian blue staining (Sigma-Aldrich) according to the manufacturer's protocol. For quantitative measurement, stained cells were extracted with $6 \mathrm{M}$ guanidine $\mathrm{HCl}$ for $2 \mathrm{~h}$ at RT and absorbance measured at $650 \mathrm{~nm}$ [14].

\section{Myogenic differentiation}

About $70 \%-80 \%$ confluence cells were cultured in myogenic differentiation medium containing low glucose DMEM (Sigma-Aldrich), 10\% FBS, 1\% P/S, and supplemented with $10 \mu \mathrm{g} / \mathrm{mL}$ insulin (Sigma-Aldrich) and $5 \mu \mathrm{g} / \mathrm{mL}$ transferrin (Sigma-Aldrich) for 2 weeks [15].

\section{HA synthetase Induction in EOM-MPCs}

The EOM-MPCs $\left(4 \times 10^{4}\right)$ were cultured in six-well plate in normal growth conditions. Cells were incubated with $1 \mu \mathrm{M}$ tafluprost for $24 \mathrm{~h}$. The expressions of hyaluronic acid synthetase (HAS) family genes were determined.

\section{Quantitation of messenger RNA expression}

Total RNA was extracted from EOM-MPCs (passage 4-6) in normal and GO using TRIzol RNA Isolation Reagent, according to the manufacturer's instructions. We used $1 \mu \mathrm{g}$ of RNA for cDNA synthesis with amfiSure Taq DNA Polymerase (GeneDEPOT, Katy, TX). The primers used for reverse transcription polymerase chain reaction (RT-PCR) are listed in Table 1 . The polymerase chain reactions (PCRs) were determined with initial activation at $95^{\circ} \mathrm{C}$ for $2 \mathrm{~min}$, followed by 35 cycles: $95^{\circ} \mathrm{C}$ for $20 \mathrm{~s}, 45^{\circ} \mathrm{C}-60^{\circ} \mathrm{C}$ for $40 \mathrm{~s}$, and $72^{\circ} \mathrm{C}$ for $40 \mathrm{~s}$. All the levels were normalized by $18 \mathrm{~S}$ ribosomal RNA expression (18s rRNA).

\section{Statistics}

Results are expressed as mean \pm standard error of the mean, and analyzed using GraphPad Prism software. The two-tailed unpaired Student's $t$-test was used to analyze normally distributed data, and the Mann-Whitney test was used to analyze nonparametric data. Statistical significance was accepted for values of $P<0.05$.

\section{Results}

\section{Characterization of EOM-MPCs from normal} subjects and $\mathrm{GO}$ patients

We used flow cytometry to characterize the surface markers of the EOM-MPCs. Our results show that the EOMMPCs strongly expressed CD44, CD59, CD73, and CD90, but weakly expressed CD34 and CD45. Although the GOEOM-MPCs expressed more CD73 than did normal EOMMPCs, and despite low-level expression of the MSC marker CD90 in GO cells, there was no significant difference between the two types of EOM-MPCs (Fig. 1A). To characterize EOM-MPCs from normal subjects and GO patients, we also examined the messenger RNA (mRNA) expression 

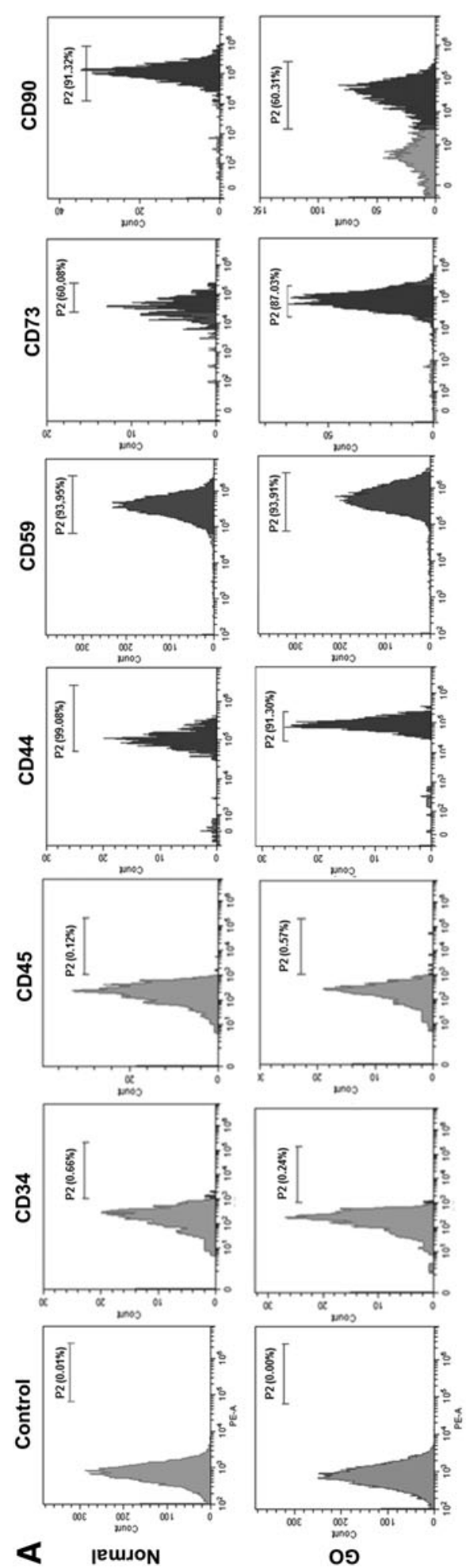

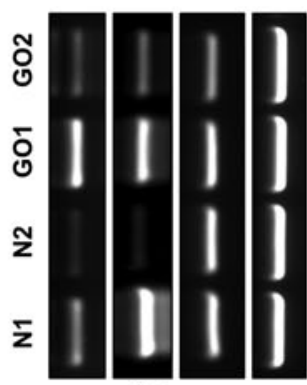

$\frac{0}{0}$

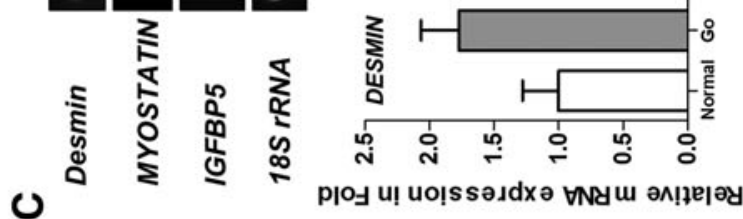

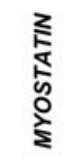

0

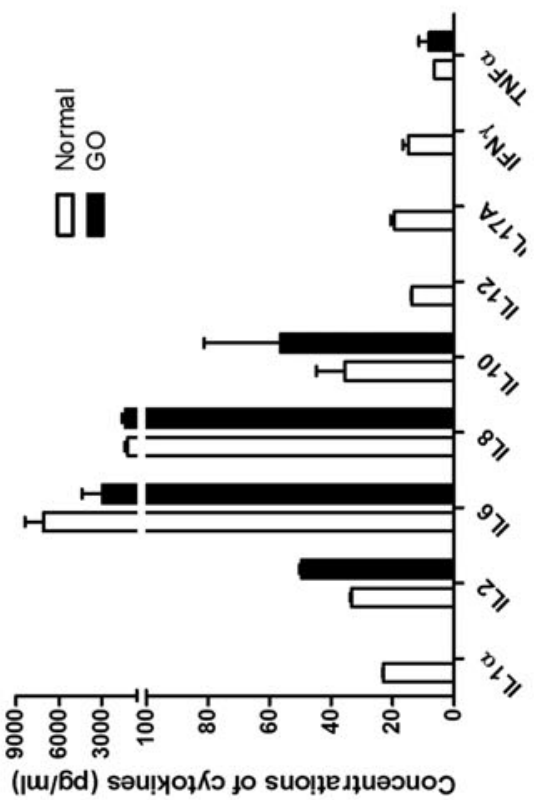

m

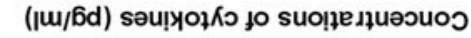

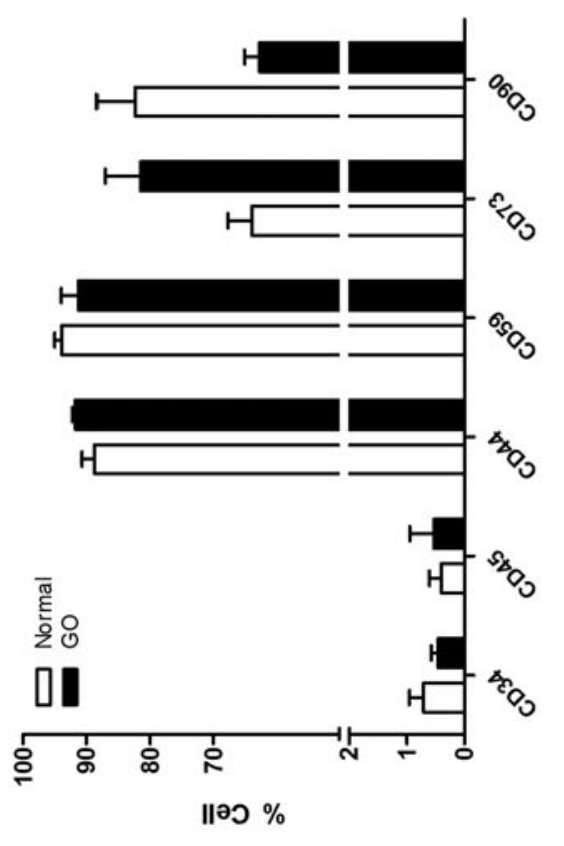

ㄱํㅇํㅇ

卷

운

o 0

흥보

交文

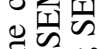

$+1$.

这

욤.

死的

政

$\varangle$

월

I

ํํ블

نे

我

$\sum_{1}^{1}$.

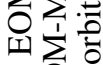

엉에

这苛

छี

迎

के 용

प艹 $\Xi$.

品

สే สี

원

웧

$3=$

그을

उ.

II

这远

نे สี

通完

政

计

प艹

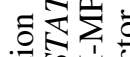

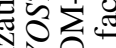

리네

之芜它

원

论苛

领远

웡

的寻

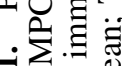

-

ن잉 
of desmin, myostatin, and insulin-like growth factor binding protein 5 (IGFBP5) (Fig. 1C). We found higher mRNA expression of desmin in GO patients compared with normal subjects, but lower expression of myostatin and IGFBP5 when compared with the same subjects (Fig. 1C).

\section{EOM-MPCs secrete variable levels of cytokines}

We evaluated 12 human cytokines in EOM-MPCs (Fig. 1B). Our results show high-level expression of IL- $1 \alpha$, IL-6, IL-8, IL-12, IL-17a, and IFN- $\gamma$, but low-level expression of surface markers such as Il-2, IL-10, and TNF- $\alpha$, in the EOM-MPCs of normal subjects compared with GO patients.

\section{Adipogenic differentiation of EOM-MPCs from normal and GO patients}

The EOM-MPCs isolated from normal subjects and GO patients were spindle-shaped cells (data not shown). The two types of EOM-MPCs were incubated with adipogenic induction medium for 14 days to examine lipid droplets, and they were compared with noninduced cells as a control. After Oil Red O staining, the induced EOM-MPCs showed significantly higher levels of staining for lipid droplets when compared with noninduced EOM-MPCs. However, the two types of EOM-MPCs showed no difference in these levels during adipogenesis (Fig. 2A). The mRNA expression of adipogenic factors such as $P P A R \gamma$ and $C / E B P \alpha$ was also not significantly different between the two types of EOM-MPCs. However, the mRNA levels of $F A B P 4$ from GO patients were significantly lower than those from normal subjects (Fig. 2B).

\section{Osteogenic differentiation of EOM-MPCs from normal and GO patients}

Both types of EOM-MPCs were cultured in osteogenic medium for 14 days. Both types of EOM-MPCs showed positive staining with Alizarin Red in osteogenic induction medium when compared with normal medium (Fig. 3A). However, quantitative staining of normal EOM-MPCs showed a significant increase under induced conditions when compared with noninduced conditions (Fig. 3A). We also determined the mRNA expression levels of factors involved in osteogenesis, including OCN, bone morphogenetic protein (BMP)-2, and SP7. In contrast to SP7 expression, the mRNA expression of $O C N$ and $B M P 2$ did not differ between normal and GO EOM-MPCs (Fig. 3B).

\section{Chondrogenic differentiation of EOM-MPCs from normal subjects and GO patients}

EOM-MPCs were cultured in chondrogenic medium for 14 days. Both types of EOM-MPCs showed positive staining with Alcian blue in chondrogenic induction medium when

A Normal medium
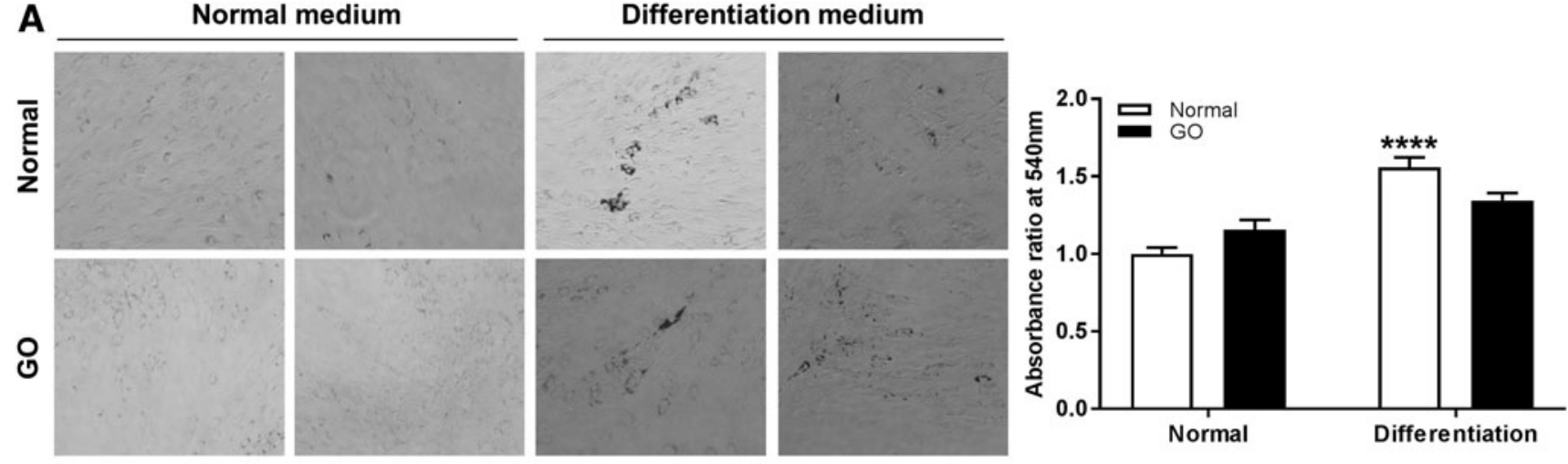

B
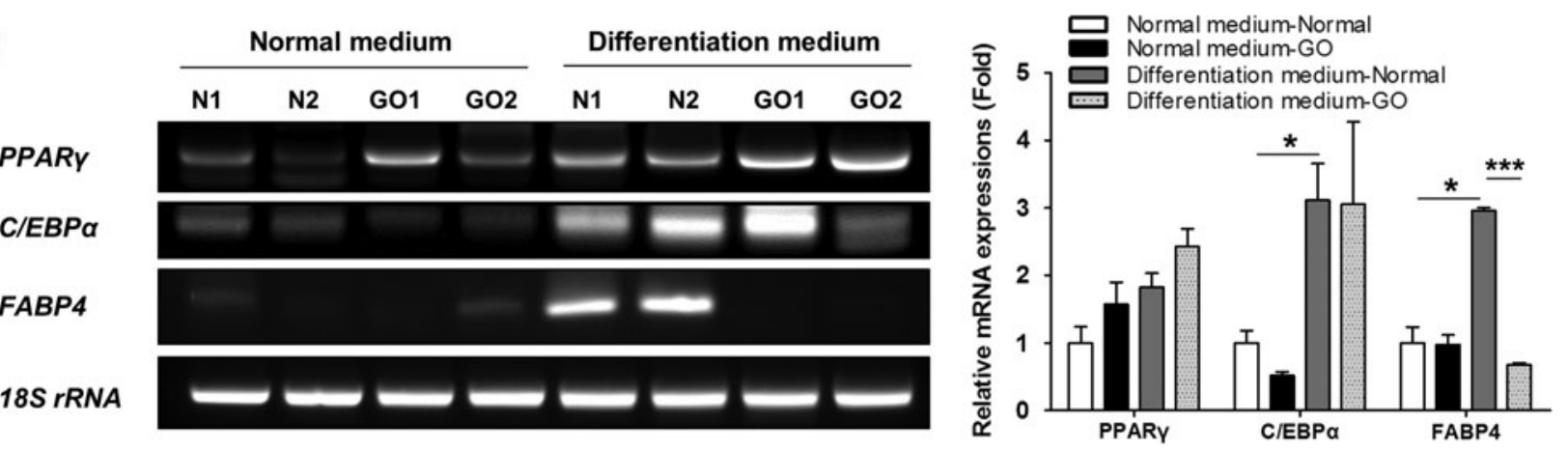

FIG. 2. Tendency of EOM-MPCs toward adipogenic differentiation. (A) Microscopic photograph of EOM-MPCs cultured in adipogenic medium for 14 days and subjected to lipid staining with Oil Red O. Control cells were grown in DMEM/F12 medium for the same period. Quantitative analysis of lipid droplet accumulation by determining the amount of dye extracted with isopropanol and measured at absorbance $540 \mathrm{~nm}$. (B) PCR analysis for genes involved in adipogenesis, such as $P P A R \gamma$, $C / E B P \alpha$, and $F A B P 4$. The mRNA expression values were presented by normalized with its noninduced cells. Results are presented as mean \pm SEM, $* * * * P<0.0001$ versus noninduced normal EOM-MPCs. $* P<0.05$ and $* * * P<0.001$. DMEM, Dulbecco's modified Eagle medium; mRNA, messenger RNA; PCR, polymerase chain reaction. 

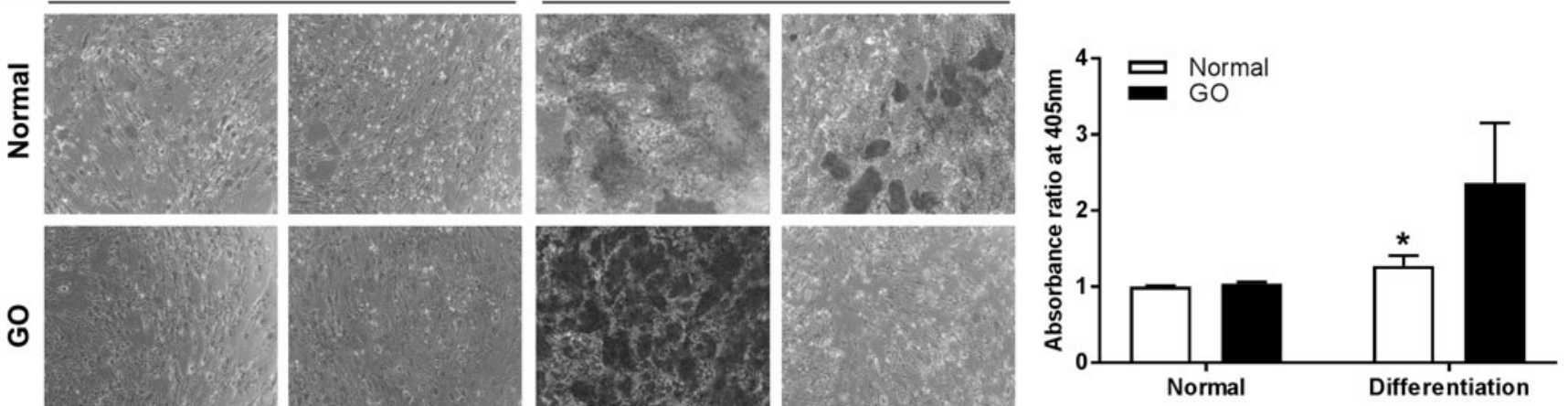

B

\begin{tabular}{|c|c|c|c|c|c|c|c|}
\hline & Norma & mediu & & Diff & rentiat & on mec & ium \\
\hline & N2 & G01 & GO2 & N1 & N2 & G01 & GO2 \\
\hline OCN & & & 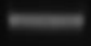 & $=$ & 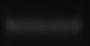 & 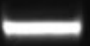 & \\
\hline BMP2 & ing & & 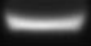 & 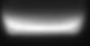 & 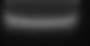 & ing & tent \\
\hline SP7 & $=$ & (a) & $=$ & 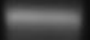 & 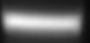 & $=$ & 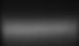 \\
\hline & $=$ & & - & 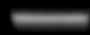 & 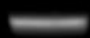 & 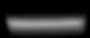 & - \\
\hline
\end{tabular}

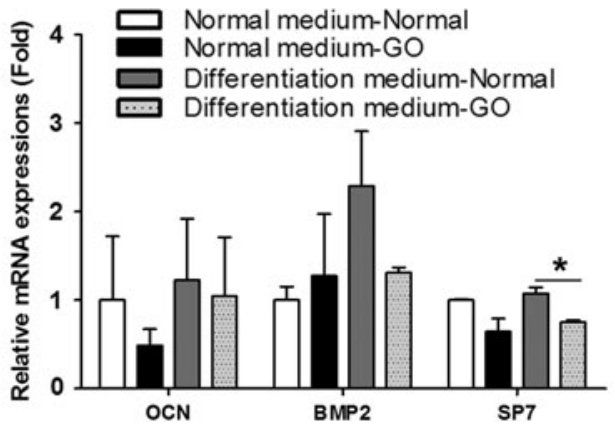

FIG. 3. Tendency of EOM-MPCs toward osteogenic differentiation. (A) Microscopic photograph of EOM-MPCs cultured in osteogenic medium for 14 days and stained with Alizarin Red staining. Control cells were grown in DMEM/F12 medium for the same period. Quantitation analysis of mineralization by determining the amount of dye extracted and measured at absorbance $405 \mathrm{~nm}$ wavelength. (B) PCR analysis for genes involved in osteogenesis, such as $O C N, B M P 2$, and $S P 7$. The mRNA expression values were presented by normalized with its noninduced cells. Results are presented as mean \pm SEM, $* P<0.05$ versus noninduced normal EOM-MPCs.

A Normal medium
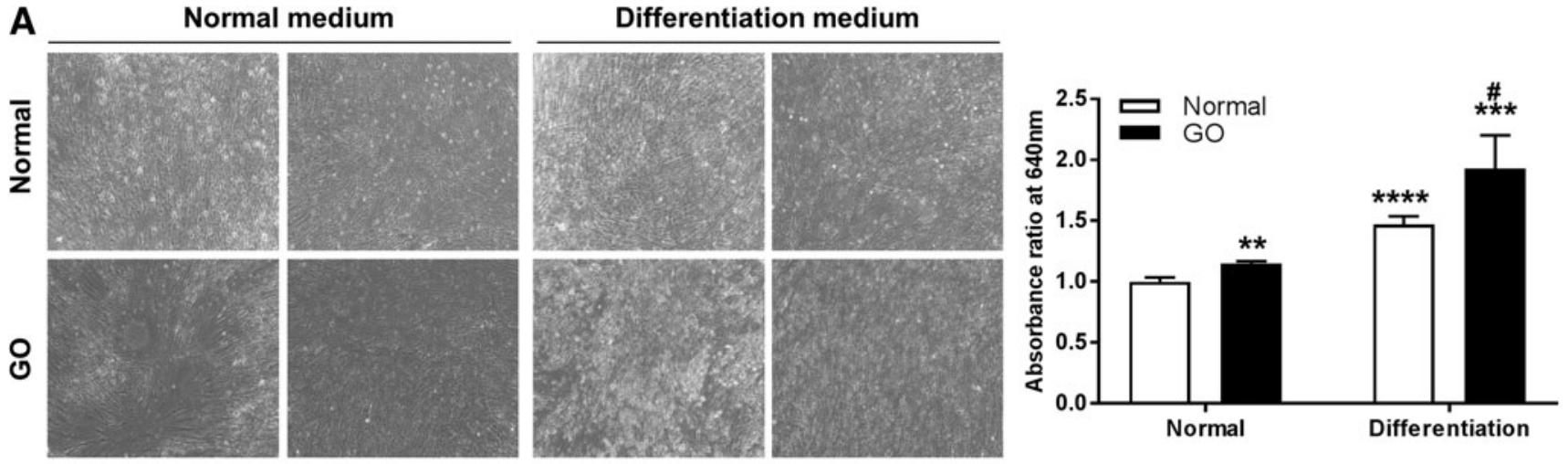

B
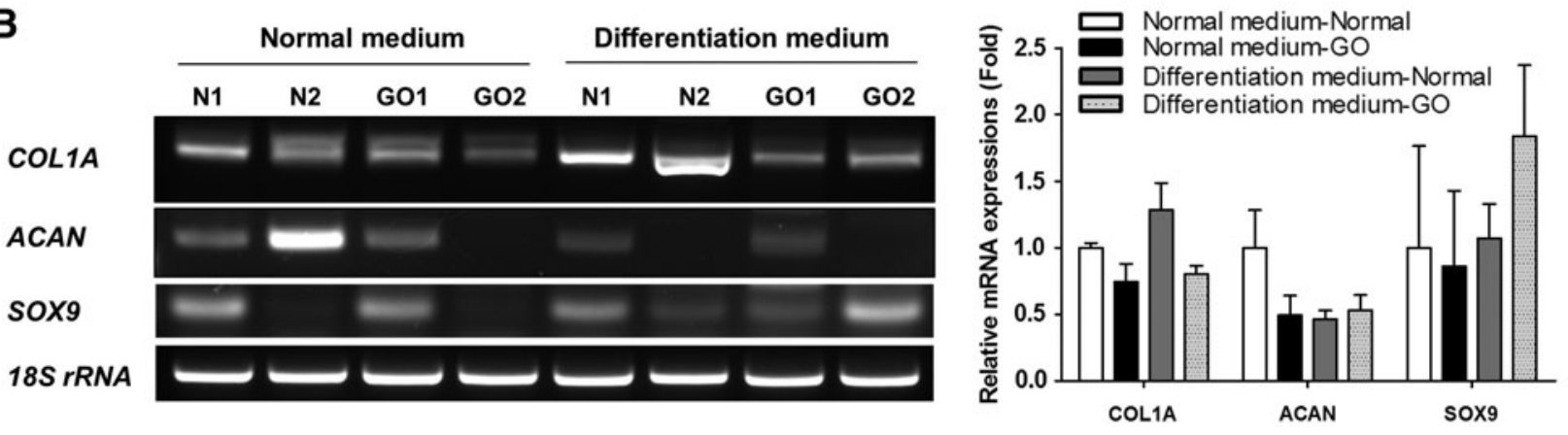

FIG. 4. Tendency of EOM-MPCs toward chondrogenic differentiation. (A) Microscopic photograph of EOM-MPCs cultured in chondrogenic medium for 14 days and stained with Alcian blue staining. Control cells were grown in DMEM/ F12 medium for the same period. Quantitation analysis of extracellular matrix production by determining the amount of dye extracted with $6 \mathrm{M}$ guanidine $\mathrm{HCl}$ and measured the absorbance at $620 \mathrm{~nm}$ wavelength. (B) PCR analysis for genes involved in chondrogenesis, such as COL1A, ACAN, and SOX9. The mRNA expression values were presented by normalized with its noninduced cells. Results are presented as mean \pm SEM, $* * P<0.005, * * * P<0.001$, **** $P<0.0001$ versus noninduced normal EOM-MPCs, ${ }^{\#} P<0.05$ versus noninduced GO EOM-MPCs. 
compared with normal medium (Fig. 4A). Our quantitative staining results show that both differentiated EOM-MPCs were significantly increased when compared with noninduced cells (Fig. 4A). Positive staining was also significantly increased in differentiated EOM-MPCs when compared with noninduced EOM-MPCs from GO patients (Fig. 4A). We also determined the mRNA expression levels of factors involved in chondrogenesis, including COL1A, ACAN, and SOX9. The expression levels of these factors did not differ between normal and GO EOM-MPCs (Fig. 4B).

\section{Myogenic differentiation of EOM-MPCs from normal subjects and GO patients}

Both EOM-MPCs were cultured in chondrogenic medium for 14 days. Quantitative staining showed that both types of differentiated EOM-MPCs were significantly increased when compared with noninduced cells (Fig. 5). We also determined the mRNA expression levels of factors involved in myogenesis, including PAX3, MYOD1, myogenin, and dystrophin. $P A X 3$ expression in GO cells was significantly lower than that in normal cells in the undifferentiated state. The mRNA expression levels of MYODI and myogenin were significantly lower in differentiated cells from GO patients than in undifferentiated cells. However, the expression of dystrophin did not differ between normal and GO EOM-MPCs (Fig. 5).

\section{HAS induction in EOM-MPCs}

The expression level of HAS1 was higher in EOM-MPCs from normal subjects when compared with GO patients under both control conditions and after induction with tafluprost. However, the expression levels of HAS2 were similar in both EOM-MPCs from normal subjects and GO patients. HAS2 induction in EOM-MPCs was significantly increased by tafluprost in normal subjects compared with GO patients. In EOM-MPCs from GO patients, TSG6 was also significantly increased under both normal conditions and after stimulation with tafluprost, when compared with EOM-MPCs from normal subjects. In addition, tafluprost treatment significantly decreased TSHR expression compared with control treatment in normal EOM-MPCs (Fig. 6).

\section{Discussion}

EOM is a specialized muscle type that controls eyeball movement and differs from other skeletal muscles [16]. Muscular dystrophies rarely involve this group of muscles, suggesting their unique quality $[17,18]$. EOM tissue can be removed during corrective strabismus surgery to adjust functioning [19] and can be used for MSC isolation. Because EOM tissue is extremely innervated compared with other skeletal muscles [3], EOM-derived MSCs have been recently reported to differentiate into neuronal lineages.

Regarding the isolation of primary myoblasts, Goetsch et al. [9] reported a method to simultaneously isolate myoblasts and fibroblasts from a single muscular tissue to establish an in vitro co-culture wound healing assay. They optimized the protocol to efficiently sort primary fibroblasts and myoblasts by serial preplating, followed by the cultivation of both cell types. Mawrie et al. [3] collected EOM tissue in vials containing DMEM with antibiotics. The

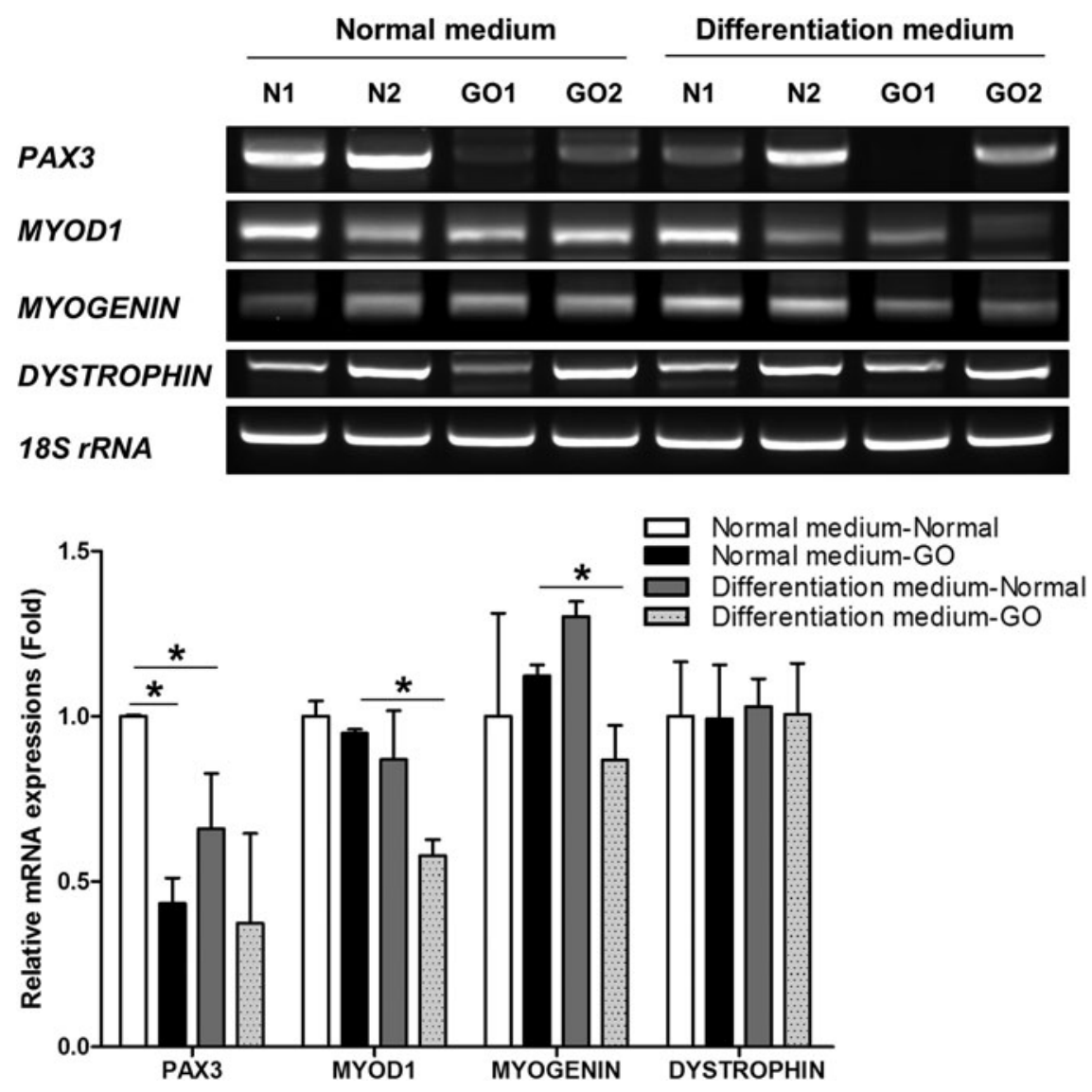

FIG. 5. Tendency of EOM-MPCs toward myogenic differentiation. PCR analysis for genes involved in myogenesis, such as $P A X 3, M Y O D 1$, MYOGENIN, and DYSTROPHIN. Results are presented as mean $\pm \mathrm{SEM}$, $* P<0.05$. 


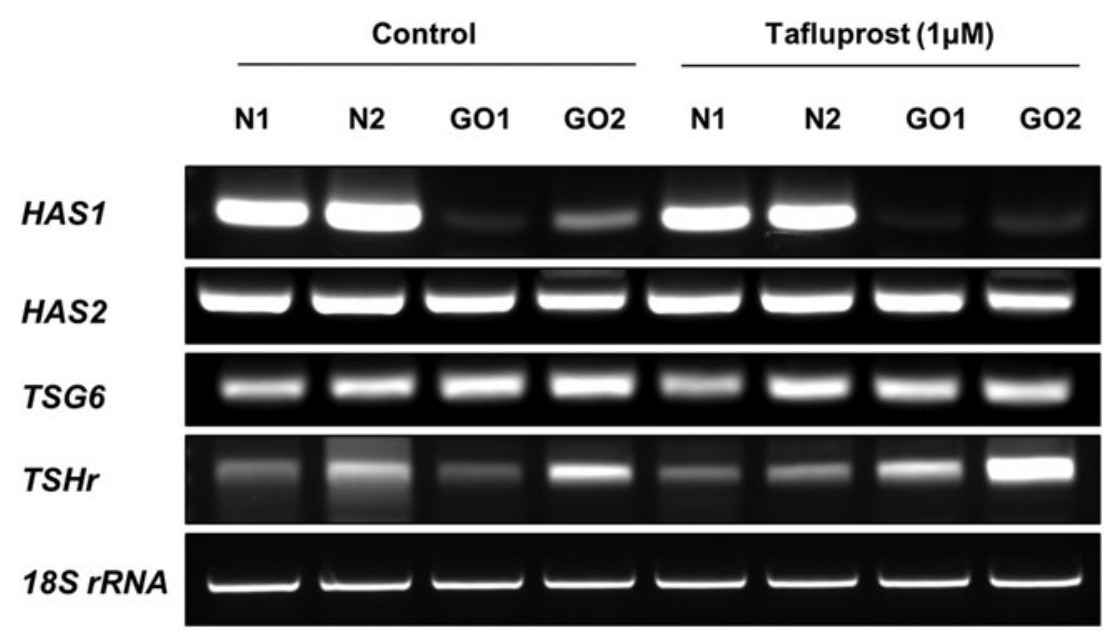

FIG. 6. HAS Induction in EOM-MPCs. PCR analysis for genes involved in HAS, such as HAS1, HAS2, TSG, and TSHR. Results are presented as mean \pm SEM, $* P<0.05$. HAS, hyaluronic acid synthetase.

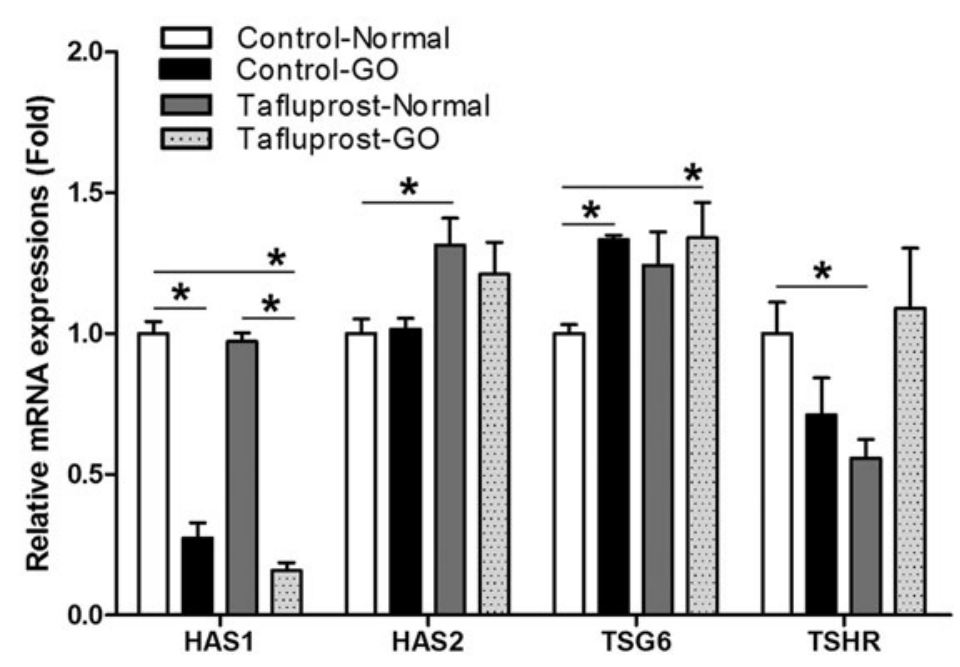

tissue was mechanically separated with forceps and plated in DMEM with $10 \%$ FBS. Fresh medium was added continuously to form colonies of spindle-shaped cells, which included CD34-positive cells.

We found that multipotent MPCs could be successfully harvested from human EOM tissues while maintaining their mesenchymal differentiation ability. The cell surface expression of CD90, CD73, CD105, and other markers along with the spindle-shaped morphology and mesenchymal differentiation capacity suggest that the EOM-MPCs resemble other mesenchymal tissue-derived MSCs [20,21]. All EOM-MPCs of the two types were positive for MSC markers. However, the patterns of CD73 and CD90 marker expression differed between the two types of EOM-MPCs. Several studies have shown that CD90 (Thy-1) functions in the fibrogenic regulation of fibroblasts as a modulator [2224]. Thy-1 knockout mice showed severe histopathological lung fibrosis [22], and a lack or blockade of thy-1 and its interacting molecules could not inhibit myofibroblastic differentiation [23,24]. However, how differences in CD marker expression between normal and GO cells regulate the potential of EOM-MPCs remains unclear.

Nonetheless, the EOM-MPCs that we isolated were pure muscle progenitors because they were negative for CD34, a previously identified marker of hematopoietic progenitor cells $[25,26]$.
Muscle injury enhances the cross-talk between satellite cells and fibroblasts [27]. Fibroblasts secrete stromal cellderived factor 1 , which induces the migration of myoblasts to a new habitat, with the migration of satellite cells to the injured site [28,29]. They also secrete hepatocyte growth factor and fibroblast growth factor, which stimulate myogenesis for muscle regeneration [30-32]. The paracrine effects of fibroblasts are therefore also considered during myoblast migration after muscle injury.

Muscle growth is regulated by factors such as growth differentiation factor 8, also known as myostatin, and IGFBP5. It is well known that myostatin modulates muscle homeostasis, and that myostatin is involved in myocyte differentiation and proliferation. A study using Lutjanus guttatus lacking myostatin resulted in muscle fiber hypertrophy, with myostatin and IGFBP5 downregulated in T3treated rats $[33,34]$. In addition, myostatin inhibition is used to treat oculopharyngeal muscular dystrophy; Dickson et al. [35] reported that target treatment for myostatin improved the body and muscle masses in patients, and reduced the levels of muscle fibrosis markers. Bostwick et al. [36] found elevated levels of IGFBP5 when investigating the fibroblasts in fibrotic lung tissue, and that the expression of IGFBP5 was increased in liver fibrosis. However, IGFBP5 was downregulated in GO thyroid tissue [37]. IGFBP5 has different functions, which are dependent on the cell and tissue type [38,39]. In this study, 
we found low-level expression of myostatin and IGFBP5 in GO EOM-MPCs compared with those from normal subjects (Fig. 1C). A comparison of EOM-MPCs and orbital fibroblasts from GO patients has not been conducted. However, identifying the relationships between regulatory factors could facilitate the development of antifibrotic therapies.

Several studies have reported that MSCs can effectively be used in various clinical settings. MSCs have been widely isolated from bone marrow, adipose tissue, and UCB [1]. UCB-derived MSCs provide the potential for skeletal myogenic differentiation as a suitable source for skeletal muscle repair and muscle-related tissue engineering [15]. MSCs from ocular limbal stroma have also demonstrated multipotent differentiation capacities [40]. In addition, EOM-MPCs were reported to have high myogenic and osteogenic differentiation capabilities, so they could also be used for repair and regeneration after tissue injury.

Regarding myogenesis, the PITX2 gene has been shown to play an integral role in limb myogenesis, as an intermediate between Pax3 and MyoD [41]. PITX2 prevents apoptosis in EOM, and it induces the myogenesis of EOM cells [42]. The specific surface chemistry of the culture plate was recently reported to enhance osteogenic and chondrogenic differentiation, and to maintain biocompatibility [43]. N-terminal modification was found to provide an optimal environment to support osteogenesis, whereas $\mathrm{COOH}$ modification promoted chondrogenesis [43]. Insulin-like growth factor-1 (IGF-1), an important regulator of cartilage and bone development, was found to be remarkably upregulated in a chondrogenesis in vitro model [14]. Real-time PCR experiments showed that $10 \mathrm{ng} / \mathrm{mL}$ of IGF- 1 and $100 \mathrm{ng} / \mathrm{mL}$ of BMP-2 were more potent than BMP-2 or IGF-1 alone, and the combination could further enhance the expression of Col2al and Sox 9 on days 3 and 6 [14].

The accumulation of glycosaminoglycans with $\mathrm{T}$ cell infiltration in fibroblasts is symptomatic of GO pathological findings in orbital fat and EOMs [2]. Enlargement of the EOMs and hypertrophy of orbital fat lead to an increased volume within the orbit, resulting in frequent GO symptoms such as orbital pain, double vision, and vision loss [2]. However, severely fibrotic EOM from GO patients decreases eye movements but produces similar EOM-MPCs as those from normal individuals in terms of the differentiation capabilities discussed in this study.

Regarding the capabilities of multilineage differentiation, there was no significant difference in the EOM-MPCs between normal subjects and GO patients, involving adipocytes, chondrocytes, osteocytes, and myocytes. However, we found that the level of HAS2 expression was higher in EOM-MPCs from GO patients than in those from normal subjects. HA production and accumulation in the fibroblasts of the EOM induce orbital tissue edema and swelling, which are the main causes of strabismus and proptosis in GO patients. A previous study reported that a COX-2 inhibitor significantly inhibited HAS3-induced HA production through a TGF- $\beta$ related pathway after TNF- $\alpha$ stimulation in GO myoblasts [6]. In addition, co-cultured macrophages can induce HA synthesis in orbital fibroblasts from GO patients, independent of IGF-1R signaling [44]. The activated orbital fibroblasts then secrete cytokines such as IL-1 $\beta$ to upregulate the production of prostaglandin E2. EOM enlargement in GO patients involves the accumulation of granular material, primarily consisting of collagen fibrils and glycosaminoglycans, such as HA. HA production is increased in orbital fibroblasts in response to various inflammatory cytokines such as TGF- $\beta$, which may be induced by multiple inflammation-related signaling pathways.

However, previous in vitro studies have shown that prostaglandin $\mathrm{F} 2 \alpha(\mathrm{PGF} 2 \alpha)$ reduced proliferation and adipogenesis in a murine cell line and in human orbital fibroblasts derived from patients with inactive GO [45]. Cases of enophthalmos developing in patients treated with bimatoprost and other PGF $2 \alpha$ analogs have been reported worldwide. However, the Brain Injury and Mechanisms of Action study has shown no improvement in proptosis in GO patients with persistent double vision (diplopia) [46]. The effect of PGF $2 \alpha$ on EOM in GO patients is still unclear. The effect of a PGF agonist in EOM-MPCs was therefore investigated in this study using EOM from GO patients. Tafluprost is a prodrug of the active substance tafluprost acid, which is a structural and functional analog of PGF2 $\alpha$. Our results indicate elevated levels of HAS2 and TSG6 in GO MPCs after treatment with tafluprost.

Although we did not identify the expression of the cell surface marker protein SSEA4, the mRNA levels of transcription factors, including OCT4, NANOG, and $S O X 2$, and genuine MSC markers such as CD73 and CD90, the expression levels of PAX3 and desmin were sufficient to identify the EOM-MPCs. We could also identify EOMderived MPCs from normal subjects that showed typical gene expression and phenotypic profiles of MSCs, along with those from GO patients. The key feature of EOMMPCs was their mesodermal differentiation capacity in vitro, which makes them suitable sources for basic studies and clinical use in the treatment of muscular disorders.

\section{Author Disclosure Statement}

The authors declare no conflicts of interest regarding the publication of this article.

\section{Funding Information}

This research was supported by grants from the Korea Health Technology R\&D Project through the Korea Health Industry Development Institute (KHIDI), funded by the Ministry of Health and Welfare, Republic of Korea (grant no. HI16C1559).

\section{References}

1. Kern S, H Eichler, J Stoeve, H Kluter and K Bieback. (2006). Comparative analysis of mesenchymal stem cells from bone marrow, umbilical cord blood, or adipose tissue. Stem Cells 24:1294-1301.

2. Park M, JP Banga, GJ Kim, M Kim and H Lew. (2019). Human placenta-derived mesenchymal stem cells ameliorate orbital adipogenesis in female mice models of Graves' ophthalmopathy. Stem Cell Res Ther 10:246.

3. Mawrie D, A Kumar, D Magdalene, J Bhattacharyya and BG Jaganathan. (2016). Mesenchymal stem cells from human extra ocular muscle harbor neuroectodermal differentiation potential. PLoS One 11:e0156697.

4. Pacheco-Pinedo EC, MT Budak, U Zeiger, LH Jorgensen, S Bogdanovich, HD Schroder, NA Rubinstein and TS 
Khurana. (2009). Transcriptional and functional differences in stem cell populations isolated from extraocular and limb muscles. Physiol Genomics 37:35-42.

5. Montanaro F, K Liadaki, J Schienda, A Flint, E Gussoni and LM Kunkel. (2004). Demystifying SP cell purification: viability, yield, and phenotype are defined by isolation parameters. Exp Cell Res 298:144-154.

6. Cheng AM, HY Yin, A Chen, YW Liu, MC Chuang, H He, S Tighe, H Sheha and SL Liao. (2016). Celecoxib and pioglitazone as potential therapeutics for regulating TGFbeta-induced hyaluronan in dysthyroid myopathy. Invest Ophthalmol Vis Sci 57:1951-1959.

7. Krafts KP. (2010). Tissue repair: the hidden drama. Organogenesis 6:225-233.

8. Velnar T, T Bailey and V Smrkolj. (2009). The wound healing process: an overview of the cellular and molecular mechanisms. J Int Med Res 37:1528-1542.

9. Goetsch KP, C Snyman, KH Myburgh and CU Niesler. (2015). Simultaneous isolation of enriched myoblasts and fibroblasts for migration analysis within a novel co-culture assay. Biotechniques 58:25-32.

10. Nepali S, M Park, H Lew and O Kim. (2018). Comparative analysis of human adipose-derived mesenchymal stem cells from orbital and abdominal fat. Stem Cells Int 2018: 3932615.

11. Chen HS, CY Hsu, YC Chang, HY Chuang, CY Long, TH Hsieh and EM Tsai. (2017). Benzyl butyl phthalate decreases myogenic differentiation of endometrial mesenchymal stem/stromal cells through miR-137-mediated regulation of PITX2. Sci Rep 7:186.

12. Niada S, LM Ferreira, E Arrigoni, A Addis, M Campagnol, E Broccaioli and AT Brini. (2013). Porcine adipose-derived stem cells from buccal fat pad and subcutaneous adipose tissue for future preclinical studies in oral surgery. Stem Cell Res Ther 4:148.

13. Jaganathan BG, B Ruester, L Dressel, S Stein, M Grez, E Seifried and R Henschler. (2007). Rho inhibition induces migration of mesenchymal stromal cells. Stem Cells 25: 1966-1974.

14. Ji YH, JL Ji, FY Sun, YY Zeng, XH He, JX Zhao, Y Yu, SH $\mathrm{Yu}$ and W Wu. (2010). Quantitative proteomics analysis of chondrogenic differentiation of C3H10T1/2 mesenchymal stem cells by iTRAQ labeling coupled with on-line two-dimensional LC/MS/MS. Mol Cell Proteomics 9: 550-564.

15. Gang EJ, JA Jeong, SH Hong, SH Hwang, SW Kim, IH Yang, C Ahn, H Han and H Kim. (2004). Skeletal myogenic differentiation of mesenchymal stem cells isolated from human umbilical cord blood. Stem Cells 22:617624.

16. Ewart JC. (1894). The development of the skeleton of the limbs of the horse, with observations on polydactyly: part II. J Anat Physiol 28:342-369.

17. Kaminski HJ, M al-Hakim, RJ Leigh, MB Katirji and RL Ruff. (1992). Extraocular muscles are spared in advanced Duchenne dystrophy. Ann Neurol 32:586-588.

18. Khurana TS, RA Prendergast, HS Alameddine, FM Tome, M Fardeau, K Arahata, H Sugita and LM Kunkel. (1995). Absence of extraocular muscle pathology in Duchenne's muscular dystrophy: role for calcium homeostasis in extraocular muscle sparing. J Exp Med 182: 467-475.

19. Ober RR, EA Palmer, AV Drack and KW Wright. (2003). Retinopathy of prematurity. In: Pediatric Ophthalmology and Strabismus. Wright KW, Spiegel PH, eds. Springer, New York, NY, pp 600-628.

20. Boxall SA and E Jones. (2012). Markers for characterization of bone marrow multipotential stromal cells. Stem Cells Int 2012:975871.

21. Gimble JM, AJ Katz and BA Bunnell. (2007). Adiposederived stem cells for regenerative medicine. Circ Res 100: 1249-1260.

22. Hagood JS, P Prabhakaran, P Kumbla, L Salazar, MW MacEwen, TH Barker, LA Ortiz, T Schoeb, GP Siegal, et al. (2005). Loss of fibroblast Thy-1 expression correlates with lung fibrogenesis. Am J Pathol 167:365-379.

23. Shentu TP, TS Huang, M Cernelc-Kohan, J Chan, SS Wong, CR Espinoza, C Tan, I Gramaglia, H van der Heyde, S Chien and JS Hagood. (2017). Thy-1 dependent uptake of mesenchymal stem cell-derived extracellular vesicles blocks myofibroblastic differentiation. Sci Rep 7:18052.

24. Zhou Y, JS Hagood, B Lu, WD Merryman and JE MurphyUllrich. (2010). Thy-1-integrin alphav beta5 interactions inhibit lung fibroblast contraction-induced latent transforming growth factor-beta1 activation and myofibroblast differentiation. J Biol Chem 285:22382-22393.

25. Joe AW, L Yi, A Natarajan, F Le Grand, L So, J Wang, MA Rudnicki and FM Rossi. (2010). Muscle injury activates resident fibro/adipogenic progenitors that facilitate myogenesis. Nat Cell Biol 12:153-163.

26. McDonald AA, MD Kunz and LK McLoon. (2014). Dystrophic changes in extraocular muscles after gamma irradiation in mdx:utrophin(+/-) mice. PLoS One 9:e86424.

27. Mathew SJ, JM Hansen, AJ Merrell, MM Murphy, JA Lawson, DA Hutcheson, MS Hansen, M Angus-Hill and G Kardon. (2011). Connective tissue fibroblasts and Tcf4 regulate myogenesis. Development 138:371-384.

28. Miller RJ, G Banisadr and BJ Bhattacharyya. (2008). CXCR4 signaling in the regulation of stem cell migration and development. J Neuroimmunol 198:31-38.

29. Ratajczak MZ, M Majka, M Kucia, J Drukala, Z Pietrzkowski, S Peiper and A Janowska-Wieczorek. (2003). Expression of functional CXCR4 by muscle satellite cells and secretion of SDF-1 by muscle-derived fibroblasts is associated with the presence of both muscle progenitors in bone marrow and hematopoietic stem/progenitor cells in muscles. Stem Cells 21:363-371.

30. Bandow K, T Ohnishi, M Tamura, I Semba and Y Daikuhara. (2004). Hepatocyte growth factor/scatter factor stimulates migration of muscle precursors in developing mouse tongue. J Cell Physiol 201:236-243.

31. Fedorov YV, NC Jones and BB Olwin. (1998). Regulation of myogenesis by fibroblast growth factors requires betagamma subunits of pertussis toxin-sensitive $G$ proteins. Mol Cell Biol 18:5780-5787.

32. Neuhaus P, S Oustanina, T Loch, M Kruger, E Bober, R Dono, R Zeller and T Braun. (2003). Reduced mobility of fibroblast growth factor (FGF)-deficient myoblasts might contribute to dystrophic changes in the musculature of FGF2/FGF6/mdx triple-mutant mice. Mol Cell Biol 23: 6037-6048.

33. Torres-Velarde J, R Llera-Herrera, L Ibarra-Castro, T GarciaGasca and A Garcia-Gasca. (2020). Post-transcriptional silencing of myostatin-1 in the spotted rose snapper (Lutjanus guttatus) promotes muscle hypertrophy. Mol Biol Rep 47:443-450.

34. Postler TS, MT Budak, TS Khurana and NA Rubinstein. (2009). Influence of hyperthyroid conditions on gene ex- 
pression in extraocular muscles of rats. Physiol Genomics 37:231-238.

35. Harish P, A Malerba, N Lu-Nguyen, L Forrest, O Cappellari, F Roth, C Trollet, L Popplewell and G Dickson. (2019). Inhibition of myostatin improves muscle atrophy in oculopharyngeal muscular dystrophy (OPMD). J Cachexia Sarcopenia Muscle 10:1016-1026.

36. Nguyen XX, L Muhammad, PJ Nietert and C FeghaliBostwick. (2018). IGFBP-5 promotes fibrosis via increasing its own expression and that of other pro-fibrotic mediators. Front Endocrinol (Lausanne) 9:601.

37. Wescombe L, H Lahooti, B Gopinath and JR Wall. (2010). The cardiac calsequestrin gene (CASQ2) is up-regulated in the thyroid in patients with Graves' ophthalmopathysupport for a role of autoimmunity against calsequestrin as the triggering event. Clin Endocrinol (Oxf) 73:522-528.

38. Hwang JR, YJ Cho, Y Lee, Y Park, HD Han, HJ Ahn, JH Lee and JW Lee. (2016). The C-terminus of IGFBP-5 suppresses tumor growth by inhibiting angiogenesis. Sci Rep 6:39334.

39. Xiao W, H Tang, M Wu, Y Liao, K Li, L Li and X Xu. (2017). Ozone oil promotes wound healing by increasing the migration of fibroblasts via PI3K/Akt/mTOR signaling pathway. Biosci Rep 37.

40. Polisetty N, A Fatima, SL Madhira, VS Sangwan and GK Vemuganti. (2008). Mesenchymal cells from limbal stroma of human eye. Mol Vis 14:431-442.

41. L'Honore A, JF Ouimette, M Lavertu-Jolin and J Drouin. (2010). Pitx2 defines alternate pathways acting through MyoD during limb and somitic myogenesis. Development 137:3847-3856.

42. Zacharias AL, M Lewandoski, MA Rudnicki and PJ Gage. (2011). Pitx2 is an upstream activator of extraocular myogenesis and survival. Dev Biol 349:395-405.
43. Griffin MF, A Ibrahim, AM Seifalian, PEM Butler, DM Kalaskar and P Ferretti. (2017). Chemical group-dependent plasma polymerisation preferentially directs adipose stem cell differentiation towards osteogenic or chondrogenic lineages. Acta Biomater 50:450-461.

44. Yang IH, GE Rose, DG Ezra and M Bailly. (2019). Macrophages promote a profibrotic phenotype in orbital fibroblasts through increased hyaluronic acid production and cell contractility. Sci Rep 9:9622.

45. Draman MS, DS Morris, S Evans, A Haridas, J Pell, R Greenwood, C Foy, P Taylor, P Pooprasert, et al. (2019). Prostaglandin F2-alpha eye drops (bimatoprost) in graves' orbitopathy: a randomized controlled double-masked crossover trial (BIMA Trial). Thyroid 29:563-572.

46. Draman MS, F Grennan-Jones, L Zhang, PN Taylor, TK Tun, J McDermott, P Moriarty, D Morris, C Lane, et al. (2013). Effects of prostaglandin F(2alpha) on adipocyte biology relevant to graves' orbitopathy. Thyroid 23:16001608.

\author{
Address correspondence to: \\ Dr. Helen Lew \\ Department of Ophthalmology \\ CHA Bundang Medical Center \\ CHA University \\ Seongnam 13496 \\ Republic of Korea \\ E-mail: eye@cha.ac.kr
}

Received for publication September 17, 2019

Accepted after revision January 18, 2020

Prepublished on Liebert Instant Online January 22, 2020 